\title{
Last Exit Times and the $Q$-Matrices of Markov Chains
}

\author{
A. O. PitTENGER
}

\section{Introduction}

Let $P(t)$ be a stochastic semigroup on a countable state space $E$, where the stochasticity may be assured by the addition of an absorbing point $\Delta$. $Q$ will denote the initial derivative matrix $p_{i j}^{\prime}(0)$ with the usual properties

$$
0 \leqq q_{i j} \text { for } i \neq j ; \quad 0 \leqq \sum_{j \neq i} q_{i j} \leqq q_{i}=-q_{i i} \leqq \infty,
$$

and any future reference to a $Q$-matrix takes (1) as given. Now if $A$ is any finite subset of $E$, we will obtain in Theorem 2 a "last-exit" decomposition of $P(t)$ relative to $A$, thereby generalizing a result given in Chung [1: II. 12]. The components involved in this last exit decomposition may be interpreted probabilistically in great detail; in particular we obtain some rather interesting relations between the $Q$-matrix and last-exit times of the process (e.g. Corollary 2 to Theorem 4).

A reversal of the decomposition is proved in Theorem 5, and the method of construction given there provides the motivation for Theorem 6 which states sufficient conditions for a $P(t)$ associated with $Q$ to be unique. Finally, the results of this paper are applied to particular $Q$-matrices with instantaneous states, thereby providing alternate proofs for results of Reuter [12] and Williams [13].

Those familiar with Chung's work on boundary theory will recognize both the notation and some of the techniques used below. This is because the analytic method used to prove Theorem 1 is merely the Laplace transform of Chung's boundary decomposition given in [2] and more recently in [3]. The author employed this version in [9] to obtain results analogous to Chung's for a general state space and recently noted that the same approach goes through without substantial change if ordinary states of the state space are used as "boundary" points.

We should note that a boundary decomposition under very weak assumptions was obtained by Lamb [7] using the Doob-Ray compactification of $E$. The assumptions made for Theorem 1 are of roughly the same generality as Lamb's, but since we will be concerned with ordinary states, we will use the one point compactification of $E$ and the sample-path analysis available in Chung [1: II. 7]. This loses the advantage of dealing with a "nearly-Hunt" process, but suffices for our purposes and provides as well some insight into the need for fictitious states. However, as a hedge against future applications, we will obtain the analytic decomposition of Theorem 1 under assumptions which permit a mixture of ordinary and fictitious states in $A$. 
During the discussions below, we will refer to $x(t)$ as a process associated with $P(t)$. By this we will have in mind the right lower semi-continuous version $x_{+}$ defined by Chung in [1: II. 7] but all of the analysis will hold equally well for any well-behaved version and in particular the nearly-Hunt process of Doob [4]. We will write $x_{+}$only when there is a positive probability that the process could be at fictitious states, an event we denote by $x_{+} \notin E$.

\section{Analytic Decomposition}

For convenience we always assume $\Delta \in A$; for explicitness we list our assumptions:

$(\alpha)$ On the ordinary states of $E, P(t)$ is standard:

$$
p_{i j}(t) \rightarrow \delta_{i j} \quad \text { as } \quad t \rightarrow 0
$$

and all states in $I=E-A$ are ordinary.

$(\beta)$ For any $i, j \in E, P_{i} \cdot(\cdot) \neq P_{j} .\left(^{\cdot}\right)$; i. e. all states are distinguishable.

$(\gamma) x(t)$ is strong-Markov for stopping times defined by first hits of sets in $E$.

(The definition of the strong-Markov property for fictitious states is a bit weaker than the usual one. Since this is not central to our purpose, we omit the details here; suffice to say that for equivalence classes of Martin exit points or for the Doob-Ray compactification, we have enough strong-Markovianess for our purpose.)

Define $F(t)$ on $I \times I$ by

$$
f_{i j}(t)=P_{i}\left(T_{A}>t, x(t)=j\right)
$$

where $T_{A}$ is the first hit of the set $A$. If

then

$$
\tau_{a} \equiv \begin{cases}T_{A} & \text { on }\left\{\omega: x\left(T_{A}-\right)=a \text { or else } x\left(T_{A}-\right) \notin A, \text { and } x\left(T_{A}\right)=a\right\} \\ \infty & \text { otherwise }\end{cases}
$$

$$
P_{i}\left(\tau_{a} \leqq s+t\right)-P_{i}\left(\tau_{a} \leqq s\right)=\sum_{I} f_{i j}(s) P_{j}\left(\tau_{a} \leqq t\right),
$$

and by [1: Theorem II. 12.4] $P_{i}\left(\tau_{a} \leqq t\right)$ has a continuous derivative $h_{\bullet}^{a}(t)$ on $[0, \infty)$ such that $h_{\bullet}^{a}(t)$ is an exit law:

$$
h_{i}^{a}(s+t)=\sum_{I} f_{i j}(t) h_{j}^{a}(s), \quad s>0 .
$$

We denote by $h^{0}(i)$ the function $P_{i}\left(T_{A}=\infty\right)$ which is invariant relative to $F(t)$ :

$$
h^{0}(i)=\sum_{j} f_{i j}(t) h^{0}(j)
$$

In addition to exit laws we will also use (bounded) entrance laws relative to $F(t)$ :

$$
0 \leqq w_{j}(s+t)=\sum_{i} w_{i}(t) f_{i j}(s), \quad t>0,
$$

with $\int_{0}^{T}\left(\sum_{i} w_{i}(t)\right) d t<\infty$ for some $T>0$. We will call an entrance law null if for all $j$, $w_{j}(t) \rightarrow 0$ as $t \rightarrow 0$. 
Finally, we use the same letter for the Laplace transform of a function, although no confusion should result:

$$
\begin{aligned}
& h_{\lambda}^{a}(i)=\int_{0}^{\infty} e^{-\lambda t} h_{i}^{a}(t) d t=h^{a}(i)-\lambda F_{\lambda} h^{a}(i),{ }^{1} \\
& w_{\lambda}(i)=\int_{0}^{\infty} e^{-\lambda t} w_{i}(t) d t
\end{aligned}
$$

and similar expressions for $p_{\lambda}(i, j)$ and $f_{\lambda}(i, j)$. Operations such as occur in (2) and the symbol $\left(w_{\lambda}, h^{a}\right)$ will denote summation over $E$ with the convention

$$
h_{\lambda}^{a}(b)=h^{a}(b)=\delta_{a b} ;
$$

$w_{\lambda}(\cdot)$ and $f_{\lambda}(\cdot, \cdot)$ will be set equal to zero if one of the states in $A$ appears as an argument, unless a specific definition to the contrary is made.

We can now state

Theorem 1. $P_{\lambda}$ may be decomposed in the form

$$
p_{\lambda}(i, j)=f_{\lambda}(i, j)+\sum_{a} \sum_{b \in A} h_{\lambda}^{a}(i) M_{\lambda}(a, b) w_{\lambda}^{b}(j)
$$

where $h_{\lambda}^{a}$ is defined in (2) and $w_{\lambda}^{b}$ is the resolvent of a bounded entrance law relative to $F_{\lambda}$ with $w_{\lambda}^{b}(c)=\delta_{b c} w^{b}(b)$ and $w^{b}(b)>0$ iff $b$ is an ordinary state. $M_{\lambda}$ is a non-negative, invertible matrix on $A \times A$ with

$$
M_{\lambda}^{-1}(a, b)=L_{a b}-\Omega(a, b)+U_{\lambda}(a, b)
$$

where $U_{\lambda}(a, b)=\lambda\left(w_{\lambda}^{a}, h^{b}\right)$, and $\Omega$ is a constant matrix with $\Omega(a, a)=0$ and $\sum \Omega(a, b) \leqq 1$. For all $b \neq a, U_{\lambda}(a, b) \leqq \Omega(a, b)$. If

then

$$
A_{0}=\left\{a: d_{\mu}(a, b) \equiv \mu\left(p_{\mu}^{a}, h^{b}\right)=\delta_{a b}\right\},^{2}
$$

$$
L_{a b}= \begin{cases}\delta_{a b} & a \notin A_{0} \\ 0 & a \in A_{0}\end{cases}
$$

and $\Omega_{a b}=0$ for all $a \in A_{0}$ and $b \in A .^{3}$

Remark. As mentioned above, this is the Laplace transform of the decomposition obtained by Chung [2] for $A$ composed of classes of points in a Martin exit boundary. The proof we present here is given in a somewhat more leisurely fashion in [9].

Proof. From the assumed strong Markov property,

$$
p_{\lambda}(i, j)=f_{\lambda}(i, j)+\sum_{a} h_{\lambda}^{a}(i) p_{\lambda}^{a}(j) .
$$

\footnotetext{
${ }^{1}$ We shall write $h^{a}(i)$ for $h_{0}^{a}(i)=\int_{0}^{\infty} h_{i}^{a}(t) d t=P_{i}\left(\tau_{a}<\infty\right)$.

${ }^{2}$ We use $p_{\mu}^{a}(\cdot)=p_{\mu}(a, \cdot)$ interchangeably.

${ }^{3}$ In Chung's terminology $A_{0}$ is the set of recurrent traps.
} 
Using the resolvent equation $P_{\lambda}=P_{\mu}+(\mu-\lambda) P_{\mu} P_{\lambda}$ for $p_{\lambda}^{a}(\cdot)$ in (5), a rearrangement yields

$$
P_{\mu}^{a}\left(\delta+(\mu-\lambda) F_{\lambda}\right)=\sum_{b}\left[\delta_{a b}+(\lambda-\mu)\left(p_{\mu}^{a}, h_{\lambda}^{b}\right)\right] P_{\lambda}^{b} .
$$

Adding and subtracting $\mu\left(p_{\mu}^{a}, h^{b}\right)$ within the brackets and using (2), we have next

$$
P_{\mu}^{a}\left(\delta+(\mu-\lambda) F_{\lambda}\right)=\sum_{b}\left[\delta_{a b}-\mu\left(p_{\mu}^{a}, h^{b}\right)+\lambda\left(p_{\mu}^{a},\left(\delta+(\mu-\lambda) F_{\lambda}\right) h^{b}\right)\right] P_{\lambda}^{b}
$$

Suppose now that $a \in A_{0}$ as defined in (4). Then after setting $\mu=1$ Eq. (6) reduces to

$$
P_{1}^{a}\left(\delta+(1-\lambda) F_{\lambda}\right)=\lambda\left(P_{1}^{a},\left(\delta+(1-\lambda) F_{\lambda} h^{a}\right) P_{\lambda}^{a}\right) .
$$

Denoting the left hand side by $w_{\lambda}^{a}$ and setting

$$
U_{\lambda}(a, b)=\lambda\left(w_{\lambda}^{a}, h^{b}\right)
$$

we have $U_{1}(a, b)=\delta_{a b}$. It is easy to check that $U_{\lambda}(a, a)>0$ for all $\lambda$ by use of (2). If

then on $E$

$$
w_{\lambda}^{a}(b)=\delta_{a b} p_{1}^{a}(a)
$$

$$
w_{\lambda}^{a}=w_{v}^{a}\left(\delta+(v-\lambda) F_{\lambda}\right)
$$

which is a reflection of the equation

$$
\left(\delta+(\mu-\lambda) F_{\lambda}\right)\left(\delta+(\lambda-v) F_{v}\right)=\delta+(\mu-v) F_{v} .
$$

For $a \notin A_{0}$ divide $(6)$ by $1-d_{\mu}(a, a)$ to obtain

$$
w_{\mu, \lambda}^{a}(j)=\sum_{b}\left[\delta_{a b}-\Omega_{\mu}(a, b)+U_{\mu, \lambda}(a, b)\right] p_{\lambda}^{b}(j),
$$

where

and

$$
\begin{aligned}
w_{\mu, \lambda}^{a}(j) & =\left(1-d_{\mu}(a, a)\right)^{-1} \cdot P_{\mu}^{a}\left(\delta+(\mu-\lambda) F_{\lambda}\right)_{j}, \\
\Omega_{\mu}(a, b) & =\left(1-\delta_{a b}\right)\left(1-d_{\mu}(a, a)\right)^{-1} d_{\mu}(a, b),
\end{aligned}
$$

$$
U_{\mu, \lambda}(a, b)=\left(1-d_{\mu}(a, a)\right)^{-1} \lambda\left(P_{\mu}^{a},\left(\delta+(\mu-\lambda) F_{\lambda}\right) h^{b}\right) .
$$

Clearly $\sum \Omega_{\mu}(a, b) \leqq 1$, and since for $\mu>\lambda$

we have

$$
\lambda\left(h^{b}+(\mu-\lambda) F_{\lambda} h^{b}\right) \leqq \mu h^{b}
$$

for $\bar{b} \neq a$ and $\mu>\lambda$.

$$
U_{\mu, \lambda}(a, b) \leqq \Omega_{\mu}(a, b),
$$

Fix $\lambda=\lambda_{0}$ and suppose $\mu$ diverged along $\left\{\mu_{k}\right\}$ in such a way that $U_{\mu_{k}, \lambda_{0}}(a, a) \rightarrow \infty$, but all other quantities converged to a finite limit. If we were to integrate $\lambda_{0} h^{a}$ on both sides of (8), divide by $U_{\mu, \lambda_{0}}(a, a)$, and then pass to the limit, we should have $d_{\lambda_{0}}(a, a)=1$. This violates $a \notin A_{0}$, and hence $\left\{\mu_{k}\right\}$ can be chosen so that all quantities on the right of (8) converge to finite limits for all $a \notin A_{0}$. Hence

$$
w_{\lambda_{0}}^{a}(j)=\sum_{b}\left[\delta_{a b}-\Omega(a, b)+U_{\lambda_{0}}(a, b)\right] p_{\lambda_{0}}^{b}(j)
$$

where $w_{\lambda_{0}}^{a}(j), \Omega$ and $U_{\lambda_{0}}$ are the obvious limits. 
Fix $j \in E$ and let $0<\lambda \neq \lambda_{0}$. From (9) we have

$$
w_{\mu, \lambda}^{a}(j)=w_{\mu, \lambda_{0}}^{a}\left(\delta+\left(\lambda_{0}-\lambda\right) F_{\lambda}\right)_{j} .
$$

Hence defining $w_{\lambda}^{a}$ by

$$
\begin{gathered}
w_{\lambda}^{a}(j)=w_{\lambda_{0}}^{a}\left(\delta+\left(\lambda_{0}-\lambda\right) F_{\lambda}\right)_{j}, \\
\sum_{E}\left|w_{\mu, \lambda}^{a}(j)-w_{\lambda}^{a}(j)\right| \leqq \\
\sum_{b}\left(\left|\Omega(a, b)-\Omega_{\mu}(a, b)\right|\right. \\
\left.+\left|U_{\mu, \lambda_{0}}(a, b)-U_{\lambda_{0}}(a, b)\right|\right)\left(\lambda_{0}+\left(\lambda_{0}-\lambda\right) / \lambda\right) .
\end{gathered}
$$

and $w_{\mu, \lambda}^{a}(j) \rightarrow w_{\lambda}^{a}(j)$ uniformly in $j \in E$ as $\mu \rightarrow \infty$ along $\left\{\mu_{k}\right\}$. This suffices to prove $U_{\lambda}(a, b)=\lambda\left(w_{\lambda}^{a}, h^{b}\right)$.

Suppose now $j=c \in A$. If $c$ is fictitious, $p_{\lambda}^{b}(c)$ is zero for all $b$, so is $w_{\mu, \lambda}^{b}(c)$ and so will be $w_{\lambda}^{b}(c)$. Suppose $c \neq a$ is ordinary. Since a factor of

$$
\mu p_{\mu}^{a}(c) /\left(1-d_{\mu}(a, a)\right)
$$

appears in the convergent $\Omega_{\mu}(a, c)$,

If $c=a$,

$$
w_{\mu, \lambda}^{a}(c)=p_{\mu}^{a}(c) /\left(1-d_{\mu}(a, a)\right) \rightarrow 0 .
$$

$$
w_{\mu, \lambda_{0}}^{a}(a)=p_{\mu}^{a}(a) /\left(1-d_{\mu}(a, a)\right)
$$

must also converge, the limit is independent of $\lambda_{0}$, and we therefore have $w_{\lambda}^{a}(b)=$ $\delta_{a b} w^{a}(a)$ as a definition consistent with (13) and with the limiting operation.

Extending $\Omega$ to $A_{0}$ and defining $L$ as in the statement of the theorem, we need only prove the invertibility of $L-\Omega+U_{\lambda}$. For this we quote

Lemma 1 [2: Lemma 15.2]. Suppose $H$ is a non-negative substochastic matrix on $A \times A$. Then $\delta-H$ is not invertible iff $H$ is stochastic on a subset $C \subset A$. If $\delta-H$ is invertible, its inverse matrix has non-negative entries.

We apply this by writing $L-\Omega+U_{\lambda}=D_{\lambda}\left(\delta-H_{\lambda}\right)$ where $D_{\lambda}(a, b)=\delta_{a b}\left(L_{a a}+\right.$ $\left.U_{\lambda}(a, a)\right)$ and

$$
H_{\lambda}(a, b)=\left(1-\delta_{a b}\right) \frac{\Omega(a, b)-U_{\lambda}(a, b)}{D_{\lambda}(a, a)} .
$$

Suppose $H_{\lambda}$ is stochastic on $C \subset A-A_{0}$. Then $U_{\lambda}(a, b)=0$ for all $b$ and all $a \in C$. Hence for $a \in C$

$$
P_{\lambda}^{a}=\sum_{c} \Omega(a, c) P_{\lambda}^{c}
$$

and this leads to a contradiction of assumption $(\beta)$. Thus $\delta-H_{\lambda}$ is invertible, and the proof is completed.

From the invertibility of $L-\Omega+U_{\lambda}$, we have

Corollary 1. If $b$ is an ordinary state,

$$
M_{\lambda}(a, b)=p_{\lambda}(a, b) / w^{b}(b)
$$

Corollary 2. Let $P(t)$ be any standard semigroup on a discrete (possibly finite) state space, and let $A$ be any finite subset of $E$. (That is all states are ordinary.) Then the resolvent $P_{\lambda}$ considered as a matrix on $A \times A$ is invertible. 
Corollary 2 is rather striking, and it is reasonable to expect a proof less involved than the one above. In fact, $L$. Pitt has pointed out that it is a simple consequence of the dominance principle, an assertion which we leave as an exercise.

The invertibility of $P_{\lambda}$ on $A \times A$ also occurs in the fundamental work of Neveu [8] as well as in Kingman's work on regenerative events [6]. In fact the representations of $P_{\lambda}$ given there are quite similar to that obtained in Theorem 1. For example when $A$ is composed of ordinary states, Kingman [6: Theorem 7] gives the matrix equation

$$
P_{\lambda}^{-1}=\lambda \delta+A+\int_{0}^{\infty}\left(1-e^{-\lambda t}\right) \mu(d t)
$$

where $A$ is a constant matrix and $\mu(d t)$ a positive matrix-valued measure. Invoking the uniqueness theorem for Laplace transforms, it is easy to show that

and

$$
A_{a b}= \begin{cases}-\Omega(a, b) / w^{a}(a) & b \neq a \\ L_{a a} / w^{a}(a) & b=a\end{cases}
$$

$$
\mu_{a b}[(t, \infty)]=\frac{1}{w^{a}(a)} \sum_{I} w_{i}^{a}(t) h^{b}(i),
$$

where $w_{\lambda}^{a}(i)$ is the transform of $w_{i}^{a}(t)$. These identifications will permit probabilistic interpretations of $A$ and $\mu$ from Proposition 5 and Theorem 4 below. Thus, for example, if $b \neq a$

$$
-w^{a}(a) A_{a b}=P_{a}[\text { first hit of } A-a \text { occurs at } b] \text {. }
$$

3.

In the proof of Theorem 1, a particular sequence $\left\{\mu_{k}\right\}$ was used to define the limits needed. For completeness we show here that the results are independent of the sequence chosen. The proof uses another result on matrices whose straightforward proof we omit.

Lemma 2. Suppose $M_{\lambda}$ is a family of invertible $n \times n$ matrices on $N \times N$, and that $N$ can be broken up into two disjoint sets:

and

$$
N_{1}=\left\{k: \lim _{\lambda \rightarrow \infty} M_{\lambda}^{-1}(k, k)<\infty\right\}
$$

$$
N_{2}=\left\{k: \lim _{\lambda \rightarrow \infty} M_{\lambda}^{-1}(k, k)=\infty\right\} .
$$

Suppose further that all other components $M_{\lambda}^{-1}(1, k)$ have finite limits as $\lambda \rightarrow \infty$, with limit zero if $k \in N_{1}$ and $1 \neq k$. Then for $1 \neq k$,

and

$$
\lim _{\lambda \rightarrow \infty}\left[M_{\lambda}^{-1}(1,1) M_{\lambda}(1, k) M_{\lambda}^{-1}(k, k)\right]=-\lim _{\lambda \rightarrow \infty} M_{\lambda}^{-1}(1, k)
$$

$$
\lim _{\lambda \rightarrow \infty} M_{\lambda}^{-1}(1,1) M_{\lambda}(1, k)=\delta_{1 k} \text {. }
$$

If fictitious states occur in $A$, a preliminary result is necessary before using Lemma 2. Since the proof is completely algebraic and is sketched in [9], we merely state 
Proposition 1. If $\left\{\mu_{k}\right\}$ were chosen so that $d_{\mu}(a, b)$ converged and if $v_{a a} \equiv 0$ while then for all $b$

$$
v_{a b} \equiv \lim _{\mu_{k}} \begin{cases}\left(\Omega(a, b)-U_{\mu_{k}}(a, b)\right)\left(1-d_{\mu_{k}}(b, b)\right) & b \notin A_{0} \\ \left(\Omega(a, b)-U_{\mu_{k}}(a, b)\right) / U_{\mu_{k}}(b, b) & b \in A_{0},\end{cases}
$$

This result permits

$$
v_{a b}=0 \text {. }
$$

Proposition 2. The decomposition given in Theorem 1 is independent of $\left\{\mu_{k}\right\}$.

Proof. $U_{\lambda} \uparrow$ with $\lambda$, and we can define

$$
N_{1}=\left\{a: U_{\infty}(a, a)<\infty\right\} .
$$

Hence $M_{\lambda}$ and $M_{\lambda}^{-1}$ satisfy the hypotheses of Lemma 2. For $a \notin A_{0}$ and $b \neq a$

$$
\lim _{\lambda} \frac{d_{\lambda}(a, b)}{1-d_{\lambda}(a, a)}=\lim _{\lambda}\left[\frac{1+U_{\lambda}(a, a)}{1+U_{\lambda}(a, a)} \frac{\sum_{c} M_{\lambda}(a, c) U_{\lambda}(c, b)}{\sum_{c} M_{\lambda}(a, c)\left(L_{c a}-\Omega(c, a)\right)}\right]=\Omega(a, b) .
$$

Similarly for $j \in E$,

$$
\lim _{\mu}\left[w_{\mu, \lambda}^{a}(j)\right]=\lim _{\mu}\left[\frac{1+U_{\mu}(a, a)}{1+U_{\mu}(a, a)} \frac{\sum M_{\mu}(a, c) w_{\lambda}^{c}(j)}{\sum_{c} M_{\mu}(a, c)\left(L_{c a}-\Omega(c, a)\right)}\right]=w_{\lambda}^{a}(j) .
$$

We record here the result that for $a \notin A_{0}$

$$
\left(1+U_{\mu}(a, a)\right)\left(1-d_{\mu}(a, a)\right) \rightarrow 1 .
$$

Another useful equation is

$$
\mu\left(w_{\lambda+\mu}^{a}, h_{\lambda}^{a}\right)=U_{\lambda+\mu}(a, b)-U_{\lambda}(a, b),
$$

from which we have

$$
\lim _{\lambda \rightarrow 0} U_{\lambda}(a, b)=0
$$

\section{Interpretation of Parameters}

The disadvantage of the Laplace transform is that much of the probabilistic content of the process is smeared over. However, motivated by Corollary 1 above, we will assume

$(\delta) A$ is composed of ordinary states,

and obtain a probabilistic meaning for all of the parameters which were obtained analytically. We begin by collecting together some useful consequences of $(\delta)$.

Proposition 3. For all $a \in A, w^{a}(a)>0, M_{\lambda}(a, b)=p_{\lambda}(a, b) / w^{b}(b)$, and

$$
p_{\lambda}^{a}(j)=\sum_{b} p_{\lambda}^{a}(b) \frac{w_{\lambda}^{b}(j)}{w^{b}(b)} .
$$

Recalling that $p_{a b}^{\prime}(0)=q_{a b}=\lim _{\lambda \rightarrow \infty} \lambda^{2} p_{\lambda}^{a}(b)$,

$$
\Omega(a, b)=w^{a}(a) q_{a b}+U_{\infty}(a, b) .
$$


Proof. The first part is immediate from $(\delta)$. Eq. (18) is trivial for $a \in A_{0}$. If $a \notin A_{0}$

and hence from (14)

$$
\begin{aligned}
\lim _{\lambda} \lambda\left(1-d_{\lambda}(a, a)\right) & =\lim _{\lambda} \frac{\left(1-d_{\lambda}(a, a)\right)}{p_{\lambda}^{a}(a)} \lambda p_{\lambda}^{a}(a) \\
& =w^{a}(a)^{-1},
\end{aligned}
$$

$$
\lim _{\lambda \rightarrow \infty} \frac{U_{\lambda}(a, a)}{\lambda}=w^{a}(a)
$$

Apply Lemma 2 to complete the proof: ${ }^{4}$

$$
\begin{aligned}
q_{a b}=\lim _{\lambda \rightarrow \infty} \lambda^{2} P_{\lambda}^{a}(b) & =\lim _{\lambda}\left[U_{\lambda}(a, a) M_{\lambda}(a, b) U_{\lambda}(b, b) \frac{\lambda^{2} w^{b}(b)}{U_{\lambda}(a, a) \cdot U_{\lambda}(b, b)}\right] \\
& =\frac{\Omega(a, b)-U_{\infty}(a, b)}{w^{a}(a)} .
\end{aligned}
$$

If $T_{A-a}$ is the first hit of $A-a$, define

$$
\bar{x}(t)= \begin{cases}x(t) & t<T_{A-a} \\ \bar{A} & t \geqq T_{A-a}\end{cases}
$$

with $\bar{\Delta}$ a new absorbing point. The $\bar{x}$ process will be useful below, and related quantities will be denoted by a supersign "-_, e.g.

and

$$
\bar{p}_{\lambda}^{a}(j)=\int_{0}^{\infty} e^{-\lambda t} P_{a}(\bar{x}(t)=j) d t=\int_{0}^{\infty} e^{-\lambda t} P_{a}\left(T_{A-a}>t, x(t)=j\right) d t
$$

$$
\bar{h}_{\lambda}^{c}(a)=\int_{0}^{\infty} e^{-\lambda t} P_{a}\left(T_{A-a}=\bar{\tau}_{c} \in d t\right),
$$

where $\bar{\tau}_{c}$ is defined analogously to $\tau_{c}$, using $T_{A-a}$ instead of $T_{A}$.

We can then relate $\bar{p}_{\lambda}$ to parameters already defined:

\section{Proposition 4.}

$$
\bar{p}_{\lambda}^{a}(a)=w^{a}(a) /\left(L_{a a}+U_{\lambda}(a, a)\right)
$$

and for $a \notin A_{0}$

$$
w^{a}(a)=\int_{0}^{\infty} \bar{p}_{a a}(t) d t
$$

For $j \in I$ and $d \neq a$

and

$$
\begin{aligned}
& \bar{p}_{\lambda}^{a}(j)=\bar{p}_{\lambda}^{a}(a) \frac{w_{\lambda}^{a}(j)}{w^{a}(a)}, \\
& \bar{h}_{\lambda}^{a}(d)=\frac{\Omega(a, d)-U_{\lambda}(a, d)}{L_{a a}+U_{\lambda}(a, a)},
\end{aligned}
$$

$$
\lim _{\lambda \rightarrow \infty} \lambda \bar{h}_{\lambda}^{a}(d)=q_{a d}
$$

\footnotetext{
${ }^{4}$ We are using the fact that under assumption $(\delta), U_{\lambda}(a, a) \uparrow \infty$ for all $a$.
} 
Proof. If $a \in A_{0}$, all of the results above are trivial. Assume $a \notin A_{0}$. For $b \neq a$ the strong Markov property justifies

as well as

$$
p_{\lambda}^{a}(b)=\sum_{c \neq a} \bar{h}_{\lambda}^{a}(c) p_{\lambda}^{c}(b)
$$

$$
\bar{p}_{\lambda}^{a}(j)=p_{\lambda}^{a}(j)-\sum_{c \neq a} \bar{h}_{\lambda}^{a}(c) p_{\lambda}^{c}(j)
$$

for $j \in I \cup a$. Multiplying (26) by $M_{\lambda}^{-1}(b, d) / w^{b}(b), d \neq a$, and summing over $b \neq a$, we have

Using (27)

$$
\bar{h}_{\lambda}^{a}(d)=\frac{\Omega(a, d)-U_{\lambda}(a, d)}{w^{a}(a)}\left[p_{\lambda}^{a}(a)-\sum_{e \neq a} \bar{h}_{\lambda}^{a}(c) p_{\lambda}^{c}(a)\right] .
$$

$$
\bar{h}_{\lambda}^{a}(d)=\bar{p}_{\lambda}^{a}(a) \frac{\left(\Omega(a, d)-U_{\lambda}(a, d)\right)}{w^{a}(a)},
$$

and Eq. (25) follows by a limiting operation. Using (28) for $\bar{h}_{\lambda}^{a}(c)$ in (27) with $j=a$ gives Eq. (21) for $\bar{p}_{\lambda}^{a}(a)$, and (24) is immediate. Finally, a combination of (24) and (27) produce the last result, Eq. (23).

The algebraic manipulations above lead to the first probabilistic interpretation of parameters defined in Section 2.

Proposition 5. For all $a$ and all $b \neq a$

If $a \notin A_{0}$,

$$
\Omega(a, b)=P_{a}\left(T_{A-a}=T_{b}<\infty\right)
$$

$$
U_{\lambda}(a, 0)=U(a, 0) \equiv \lambda\left(w_{\lambda}^{a}, h^{0}\right)=P_{a}\left(T_{A-a}=\infty\right),
$$

where we recall that $h^{0}(i)=P_{i}\left(T_{A}=\infty\right)$.

Proof. For $a \in A_{0}(29)$ is immediate. For $a \notin A_{0}(29)$ follows from (16) and (24):

$$
\Omega(a, b)=\lim _{\lambda \rightarrow 0} \int_{0}^{\infty} e^{-\lambda t} d P\left(T_{A-a}=T_{b} \in d t\right)
$$

To prove (30), go back to the definition of $\Omega$ :

$$
\sum_{b} \Omega(a, b)=\lim _{\lambda \rightarrow \infty} \frac{1-d_{\lambda}(a, a)-d_{\lambda}(a, 0)}{1-d_{\lambda}(a, a)}=1-\lim _{\lambda \rightarrow \infty} \frac{\sum_{c} M_{\lambda}(a, c) U_{\lambda}(c, 0)}{1-d_{\lambda}(a, a)}
$$

and use Lemma 2 and the invariance of $h^{0}$. [ ]

\section{5.}

A deeper insight requires inverting the Laplace transform $w_{\lambda}^{a}$. This inversion is justified by

Proposition 6. $w_{\lambda}^{a}$ is the Laplace transform of an entrance law $w(t)$ on I which is continuous and bounded in the sense of Section 2. $w^{a}(t)$ may be written as

$$
w_{j}^{a}(t)=w^{a}(a)\left[\sum_{1 \in I} w^{a}(1) f_{1 j}(t)+v_{j}^{a}(t)\right]
$$


where $v^{a}(t)$ is a null entrance law and

$$
\lim _{t \rightarrow 0} w_{j}^{a}(t)=w^{a}(a) \cdot{ }_{0} w^{a}(j) .
$$

Proof. All of the assertions are contained in Neveu [8]; we have separated out the factor of $w^{a}(a)$ for convenience below.

Corollary. For all $a \in A$ and $t>0$

$$
\lim _{\varepsilon \rightarrow 0} \sum_{I} \frac{p_{a j}(\varepsilon) f_{j k}(t)}{\varepsilon}=\frac{w_{t}^{a}(k)}{w^{a}(a)}
$$

Proof. By virtue of Proposition 6 we can unwind the Laplace transform and express (17) as

$$
p_{a j}(s)=\sum_{b} \int_{0}^{s} \frac{p_{a b}(u)}{w^{b}(b)} w_{j}^{b}(s-u) d u .
$$

Hence, the left hand side of $(31)$ is

$$
\lim _{\varepsilon \rightarrow 0} \sum_{b} \int_{0}^{\varepsilon} \frac{p_{a b}(u)}{\varepsilon} \frac{w_{k}^{b}(\varepsilon+t-u) d u}{w^{b}(b)},
$$

and the right hand side is the limiting expression. [

6.

The decomposition now begins to look suspiciously like a last exit decomposition, and to make this explicit, we will use some of the technical ideas of Chung [2: II. 12], where a last-exit decomposition from one state is obtained. The main difference in the approach here is that we already have the $w^{a}(t)$ in hand and merely wish to confirm their meaning.

First of all we state:

Proposition 7. Let $i \in E, j \in I, b \in A$, and $t>0$ be fixed and let $\gamma$ be a random variable satisfying the following conditions on $r \in \mathscr{F}\{x(s): 0 \leqq s \leqq t\}$ :

(a) $0<\gamma<t$

(b) $Y=\{x(0)=i\} \cap\{x(t)=j\} \cap \Lambda$, where $\gamma$ and $\Lambda$ are related by

$$
\{\gamma>u\} \cap A \in \mathscr{F}\{x(s) ; u \leqq s \leqq t\}
$$

(c) $\underline{\lim }_{s^{\uparrow}{ }^{\prime}} x(s, w)=b .^{5}$

If $\gamma^{(m)}$ is the discrete skeleton of $\gamma$ defined by

$$
\gamma^{(m)}=\frac{n}{2^{m}} \text { for } \quad \frac{n}{2^{m}}<\gamma \leqq \frac{n+1}{2^{m}}, \quad n \geqq 0,
$$

then

$$
\lim _{m \rightarrow \infty} P_{i}\left(x\left(\gamma^{(m)}\right)=b, \Lambda\right)=P_{i}(\Lambda) .
$$

${ }^{5}$ This presupposes an order on $E$; we are using in fact the sample path analysis of Chung [2: II. 7 ] with this notation. 
Proof. This is a slight generalization of [2: II. Theorem 12.1] and the indicated property of $\Lambda$ allows the proof given there to be applied without any essential change. $\square$

For any finite set $C \subset E$, define

$$
\gamma_{C}^{t}=\left\{\begin{array}{l}
\sup (s \leqq t ; x(s) \in C) \\
0 \text { if }()=\phi .
\end{array}\right.
$$

Since we are working with a finite number of ordinary states,

$$
\left\{\gamma_{C}^{t}<t\right\}=\bigcup_{b}\left\{\gamma_{A}^{t}=\gamma_{b}^{t}<t\right\}
$$

Proposition 8. For $j \in I$

$$
P_{a}\left(x(t)=j ; \gamma_{b}^{t}=\gamma_{A}^{t}\right)=\int_{0}^{t} p_{a b}(u) \frac{w_{j}^{b}(t-u)}{w^{b}(b)} d u .
$$

Proof. As in [1: II. Theorem 12.2], we start by writing

where

$$
\sum_{v=1}^{\left[2^{m} t\right]} P_{a}\left(A_{v}\right)=\sum_{v=1}^{\left[2^{m} t\right]} p_{a b}\left(\frac{v-1}{2^{m}}\right) \sum_{I} p_{b i}\left(2^{-m}\right) f_{i j}\left(t-\frac{v}{2^{m}}\right),
$$

$$
A^{v}=\left\{x\left(\frac{v-1}{2^{m}}\right)=b ; x(s, \omega) \notin A \text { for } \frac{v}{2^{m}} \leqq s \leqq t, x(t)=j\right\} .
$$

( $\left[2^{m} t\right]$ denotes the largest integer in $2^{m} t$.) By virtue of the Corollary to Proposition 6 , the quantity on the right above converges to the right side of (32) as $m \rightarrow \infty$. If $A \equiv\left\{\gamma_{b}^{t}=\gamma_{A}^{t}\right\}$ and $\gamma_{b}^{t(m)}$ is as in Proposition 7, then with $x(0)=a$

and thus

$$
B \equiv\left\{\omega: x\left(\gamma_{b}^{t(m)}, \omega\right) \neq b ; \gamma_{b}^{t} \leqq \frac{\left[2^{m} t\right]}{2^{m}}, A, x(t, \omega)=j\right\}
$$

$$
\begin{aligned}
P_{a}\left(x(t)=j, \gamma_{b}^{t}=\gamma_{A}^{t}\right)= & \sum_{\nu=1}^{\left[2^{m} t\right]} P_{a}\left(A_{v}\right)+P_{a}(B) \\
& +P_{a}\left(\frac{\left[2^{m} t\right]}{2^{m}}<\gamma_{b}^{t}, A, x(t)=j\right) \\
& -P_{a}\left(x(t)=j, x\left(\gamma_{b}^{t(m)}\right)=b, 0<\gamma_{A}^{t}-\gamma_{b}^{t} \leqq 2^{-m}\right) .
\end{aligned}
$$

The proof is completed by noting that as $m \rightarrow \infty, P_{a}(B) \rightarrow 0$ by the preceding result, while $P_{a}\left(\gamma_{A}^{t}=t, x(t)=j\right)=0$ and $P_{a}\left(0<\gamma_{A}^{t}-\gamma_{b}^{t} \leqq 2^{-m}\right)$ also converges to zero.

All of the machinery is now in hand for an interpretation of Theorem 1 under assumption $(\delta)$ :

Theorem 2. Denote $\sum_{I} w_{i}^{b}(t)$ by $w_{I}^{b}(t)$. Then

$$
P_{a}\left(\gamma_{b}^{t}=\gamma_{A}^{t} \in d s ; x(t)=j\right)=p_{a b}(s) \frac{w_{j}^{b}(t-s)}{w^{b}(b)} d s
$$

and

$$
P_{a}\left(\gamma_{b}^{t}=\gamma_{A}^{t} \in d s\right)=p_{a b}(s) \frac{w_{I}^{b}(t-s)}{w^{b}(b)} d s .
$$


A version of the conditional probability below is

and therefore

$$
P_{a}\left(x(t)=j \mid \gamma_{b}^{t}=\gamma_{A}^{t}=s\right)=\frac{w_{j}^{b}(t-s)}{w_{I}^{b}(t-s)},
$$

$$
p_{a j}(t)=\sum_{A} \int_{0}^{t} \frac{p_{a b}(s) w_{I}^{b}(t-s)}{w^{b}(b)} \frac{w_{j}^{b}(t-s)}{w_{I}^{b}(t-s)} d s
$$

is an expression of the last-exit decomposition

$$
p_{a j}(t)=\sum_{A} \int_{0}^{t} E_{a}\left[\left\{\gamma_{b}^{t}=\gamma_{A}^{t} \in d s\right\} ; \quad P_{a}\left(x(t)=j \mid \gamma_{b}^{t}=\gamma_{A}^{t}=s\right)\right] d s .
$$

Proof. An application of Proposition 8 for $s<t$ gives

$$
\begin{aligned}
P_{a}\left(\gamma_{b}^{t}=\gamma_{A}^{t}<s ; x(t)=j\right) & =\sum_{1} P_{a}\left(\gamma_{a}^{s}=\gamma_{A}^{s}, x(s)=1, x(u) \in I \text { for } s \leqq u \leqq t ; x(t)=j\right) \\
& =\sum_{1 \in I} \int_{0}^{s} p_{a b}(u) \frac{w_{1}^{b}(s-u)}{w^{b}(b)} f_{1 j}(t-s) d u \\
& =\int_{0}^{s} p_{a b}(u) \frac{w_{1}^{b}(t-u)}{w^{b}(b)} d u .
\end{aligned}
$$

(33) and hence (34) are immediate consequences. Furthermore,

$$
P_{a}\left(\gamma_{b}^{t}=\gamma_{A}^{t}<s, x(t)=j\right)=\int_{0}^{s} P_{a}\left(\gamma_{b}^{t}=\gamma_{A}^{t} \in d u\right) \frac{w_{j}^{b}(t-u)}{w_{I}^{b}(t-u)},
$$

which suffices to prove both statements about conditional probabilities. $]$

By using (23) and a repetition of the analysis of Proposition 8 and Theorem 2, we have

Corollary. Let $\bar{x}(t)$ be the process starting at a and stopped at the first hit of $A-a$. Then

$$
\begin{array}{r}
P_{a}\left(\bar{\gamma}_{a}^{t} \in d s, \bar{x}(t)=j\right)=\bar{p}_{a a}(s) \frac{w_{j}^{a}(t-s)}{w^{a}(a)} d s, \\
P_{a}\left(\bar{\gamma}_{a}^{t} \in d s\right)=\bar{p}_{a a}(s) \frac{w_{l}^{a}(t-s)}{w^{a}(a)} d s,
\end{array}
$$

and hence a version of the conditional probability below is

$$
\frac{w_{j}^{a}(t-s)}{w_{I}^{a}(t-s)}=P_{a}\left(\bar{x}(t)=j \mid \bar{\gamma}_{a}^{t}=s\right) .
$$

It seems odd at first glance to have $w_{j}^{a}(t-s) / w_{I}^{a}(t-s)$ represent conditional probabilities for both $x(t)$ and $\bar{x}(t)$. However, since in both cases the conditioning is that the process will not hit $A$ in $(s, t]$, the result is quite reasonable. 


\section{7.}

We may apply the results above to obtain a rather detailed analysis for the influence of the $Q$-matrix of $P(t)$. We use $\gamma_{a}^{s}$ and $\gamma_{A}^{s}$ as before and define

$$
\zeta_{A}^{a}=\left\{\begin{array}{l}
\sup \left\{t<T_{A-a}: x(t)=a\right\} \\
0 \quad \text { if }\{\} \text { is empty }
\end{array}\right.
$$

Theorem 3. Suppose $a \notin A_{0}$. Then for $b \neq a$

$$
P_{a}\left(\zeta_{A}^{a} \in d s, T_{b}=T_{A-a} \in d t, \zeta_{A}^{a}<T_{A-a}\right)=\frac{\bar{p}_{a a}(s)}{w^{a}(a)} \theta_{a b}(t-s)
$$

where

$$
\theta_{a b}(t-s) \equiv \sum_{j} w_{j}^{a}(t-s-r) h_{j}^{b}(r)
$$

is independent of $0<r<t-s$. Furthermore

$$
U_{\infty}(a, b)=\int_{0}^{\infty} \theta_{a b}(v) d v=P_{a}\left(\zeta_{A}^{a}<T_{b}=T_{A-a}<\infty\right),
$$

and hence

$$
w^{a}(a) q_{a b}=P_{a}\left(\zeta_{A}^{a}=T_{b}<\infty\right) .
$$

Proof. First of all

$$
\begin{aligned}
P_{a}\left(\zeta_{A}^{a}<s<\right. & \left.t<T_{A-a}=T_{b}<\infty\right) \\
= & \sum_{1} P_{a}\left(\gamma_{a}^{s}<s<T_{A-a} ; x(s)=1 ; s<t<T_{A-a}=T_{b}<\infty\right) \\
= & \sum_{1} P_{a}\left(\bar{\gamma}_{a}^{s}<s, \bar{x}(s)=1\right) P_{1}\left(t-s<T_{A}=T_{b}<\infty\right) \\
= & \int_{0}^{s} \frac{\bar{p}_{a a}(u)}{w^{a}(a)} \sum_{1, j} w_{1}^{a}(s-u) f_{1 j}(t-s) P_{j}\left(\tau_{b}<\infty\right) d u \\
= & \int_{0}^{s} \frac{\bar{p}_{a a}(u)}{w^{a}(a)} \int_{t}^{\infty} \theta_{a b}(v-u) d v d u
\end{aligned}
$$

from which (36) follows. Eq. (37) follows from the definitions and by integrating (36), while (38) is a consequence of Proposition 5.

The reader familiar with Chung's work in boundary theory will recognize the interpretation of $q_{a b}$ above. (38) shows that with the additional factor of $w^{a}(a), q_{a b}$ is a measure of first hitting $A-a$ by a direct jump from $a$ to $b$. We can make (38) more familiar by assuming $a, b \in A_{n} \uparrow E$ and assuming $a$ is a stable state. If $\tau$ is the first hit of $E-a$, we get in the limit

or

$$
q_{a b}=P_{a}(x(\tau+)=b) / \int_{0}^{\infty} P_{a}(\tau>t, x(t)=a) d t
$$

$$
\frac{q_{a b}}{q_{a}}=P_{a}(x(\tau+)=b) .
$$


The limitation on the role of $Q$ is also apparent. If for example $P(t)$ were the semigroup constructed by Feller and McKean [5] with all instantaneous states and $q_{i j}$ zero for $i \neq j$, then there is no probability of going directly from $a$ to $b$. This is of course a reflection of the continuity of the sample paths on the completed state space and is merely the statement that between two given rationals there is a third rational.

Recall now the representation of the entrance laws guaranteed by Proposition 6. For $j \in I$

$$
\mu^{2} P_{\mu}^{a}(j)=\sum_{b} \mu p_{\mu}^{a}(b) \cdot \frac{\mu w_{\mu}^{b}(j)}{w^{b}(b)}
$$

and a passage to the limit gives

$$
q_{a j}={ }_{0} w^{a}(j)
$$

Hence, the $Q$-matrix appears in a component of $w^{a}(t)$, and this leads to the question of its probabilistic meaning.

Theorem 4. Let $\zeta_{A}^{a}$ be the last hitting time of a before $T_{A-a}$, as above, and let $j, 1 \in I$. Then ${ }^{6}$

(a) $P_{a}\left(\gamma_{b}^{t}=\gamma_{A}^{t}<s, x_{+}\left(\gamma_{b}^{t}\right)=1, x_{+}(t)=j\right)=\int_{0}^{s} p_{a b}(u) q_{b 1} f_{1 j}(t-u) d u$

and

$$
P_{a}\left(\bar{\gamma}_{a}^{t}<s, \bar{x}_{+}\left(\bar{\gamma}_{a}^{t}\right)=1, \bar{x}_{+}(t)=j\right)=\int_{0}^{s} \bar{p}_{a a}(u) q_{a 1} f_{1 j}(t-u) d u .
$$

(b) $P_{a}\left(\zeta_{A}^{a} \in d s, x_{+}\left(\zeta_{A}^{a}\right)=1, T_{A-a}=T_{b} \in d t\right)=\bar{p}_{a a}(s) q_{a 1} h_{1}^{a}(t-s) d t d s$

and for $a \notin A_{0}$

$$
P_{a}\left(\zeta_{A}^{a} \in d s, x_{+}\left(\zeta_{A}^{a}\right)=1, T_{A-a}=\infty\right)=\bar{p}_{a a}(s) q_{a 1} h^{0}(1) d s .
$$

Proof. The method follows that of Proposition 8 by showing

$$
P_{a}\left(\gamma_{b}^{t}=\gamma_{A}^{t}, x_{+}\left(\gamma_{b}^{t}\right)=1, x_{+}(t)=j\right)=\lim _{m} \sum_{v=1}^{\left[2^{m} t\right]} p_{a b}\left(\frac{v-1}{2^{m}}\right) p_{b 1}\left(2^{-m}\right) f_{1 j}\left(t-\frac{v}{2^{m}}\right)
$$

which is the desired expression. Since $\left\{\gamma_{b}^{t}=\gamma_{A}^{t}, x_{+}\left(\gamma_{b}^{t}\right)=1\right\}$ satisfies the requirements of $A$ in Proposition 7, the argument proceeds as before, except that we must consider a term of the form

$$
\lim _{m} P_{a}\left[x_{+}\left(\gamma_{b}^{t(m)}\right)=b ; x_{+}\left(\gamma_{b}^{t}\right)=1, x_{+}\left(\gamma_{b}^{t(m)}+2^{-m}\right) \neq 1, x(t)=j\right] .
$$

To show this contributes nothing in the limit, let

$$
P_{k}\left(T_{1} \leqq s\right)=\int_{0}^{s} h_{k}^{1}(v) d v
$$

\footnotetext{
${ }^{6}$ Recall the discussion of $x_{+}$in Section 1.
} 
where $h^{1}(v)$ is the continuous function on $[0, \infty)$ guaranteed by [1: Theorem II. 12.4]. Consequently the unwanted term above is bounded by

$$
\begin{aligned}
\lim _{m} \sum_{v} p_{a b}\left(\frac{v-1}{2^{m}}\right) \int_{0}^{2-m} & h_{b}^{1}(s)\left(1-p_{11}\left(2^{-m}-s\right)\right) d s \\
& \leqq \int_{0}^{t} p_{a b}(s) h_{b}^{1}(0+) \lim _{m}\left(\sup _{v \leqq 2^{-m}}\left(1-p_{11}(v)\right)\right)=0 .
\end{aligned}
$$

A repetition of the arguments in Theorem 2 then justifies part (a) above.

Proceeding to (b), the method is straightforward:

$$
\begin{aligned}
P_{a}\left(\zeta_{A}^{a}<s\right. & \left.<t<T_{A-a}=T_{b}<\infty, x_{+}\left(\zeta_{A}^{a}\right)=1\right) \\
& =\sum_{j} P_{a}\left(\gamma_{a}^{s}<s<T_{A-a}, x_{+}\left(\gamma_{a}^{s}\right)=1, x(s)=j\right) P_{j}\left(t-s<T_{A}=\tau_{b}<\infty\right) \\
& =\int_{0}^{s} \bar{p}_{a a}(u) q_{a 1} \int_{t}^{\infty} h_{1}^{b}(v-u) d v d u
\end{aligned}
$$

and (42) is immediate. Eq. (43) follows by an obvious modification of the preceding argument and the invariance of $h^{0}$.

As immediate consequences, we have

Corollary 1. For $s<t$

$$
\frac{w^{b}(b) q_{b 1} f_{1 j}(t-s)}{w_{I}^{b}(t-s)}=P_{a}\left(x_{+}(t)=j, x_{+}\left(\gamma_{A}^{t}\right)=1 \mid \gamma_{b}^{t}=\gamma_{A}^{t}=s\right) .
$$

Hence

$$
\frac{w^{b}(b) \sum_{1} q_{b 1} f_{1 j}(t-s)}{w_{I}^{b}(t-s)}=P_{a}\left(x_{+}(t)=j, x_{+}\left(v_{A}^{t}\right) \in I \mid \gamma_{b}^{t}=\gamma_{A}^{t}=s\right),
$$

and

$$
\frac{w^{b}(b) v_{j}^{b}(t-s)}{w_{I}^{b}(t-s)}=P_{a}\left(x(t)=j, x_{+}\left(\gamma_{A}^{b}\right) \notin I \mid \gamma_{b}^{t}=\gamma_{A}^{t}=s\right) .
$$

Proof. (44) follows from (34) in Theorem 2 and (40) above by a now standard argument. The remainder of the Corollary is then immediate.

We next summarize (38) and the integrals of (42) and (43) above in

Corollary 2. Suppose $a \notin A_{0}$. Then for all $1 \in E-a$ and $b \neq a$

and

$$
P_{a}\left(x_{+}\left(\zeta_{A}^{a}\right)=1, T_{A-a}=T_{b}<\infty\right)=w^{a}(a) q_{a 1} h^{b}(1),
$$

For $1 \in I$

$$
P_{a}\left(x_{+}\left(\zeta_{A}^{a}\right) \notin E-a, T_{A-a}=T_{b}<\infty\right)=w^{a}(a) \lim _{t \rightarrow 0}\left(V^{a}(t), h^{b}\right) .
$$

and

$$
P_{a}\left(x_{+}\left(\zeta_{A}^{a}\right)=1, \zeta_{A}^{a}<\infty=T_{A-a}\right)=w^{a}(a) q_{a 1} h^{0}(1),
$$

$$
P_{a}\left(x_{+}\left(\zeta_{A}^{a}\right) \notin I, \zeta_{A}^{a}<\infty=T_{A-a}\right)=w^{a}(a) \lim _{t \rightarrow 0}\left(V^{a}(t), h^{0}\right) .
$$

Proof. The second and fourth equations follow from the interpretation of $\Omega(a, b)$ and Eq. $(30)$. 
Finally we have another expression for $w^{a}(a)$, and we include a striking relationship between $q_{a}$ and $V^{a}(t)$.

Corollary 3. For $a \notin A_{0}$

$$
\left(w^{a}(a)\right)^{-1}=\sum_{k} q_{a k}\left(1-h^{a}(k)\right)+\lim _{t \rightarrow 0}\left(v^{a}(t),\left(1-h^{a}\right)\right) .
$$

If $q_{a}-\sum_{k \neq a} q_{a k} \geqq 0$ for any $a \in A$, then

$$
q_{a}=\sum_{k \neq a} q_{a k}+\lim _{t \rightarrow 0}\left(v^{a}(t), 1\right) .
$$
we have

Proof. (47) follows from $1=\sum_{b} \Omega(a, b)+U(a, 0)$ and Corollary 2. To prove (48)

$$
\begin{aligned}
q_{a} & =\lim _{\lambda} \lambda\left(1-\lambda p_{\lambda}(a, a)\right)=\lim _{\lambda} \lambda \sum_{b} \cdot p_{\lambda}^{a}(b)\left(\frac{L_{b a}-\Omega(b, a)+U_{\lambda}(b, a)}{w^{a}(a)}-\lambda \delta_{b a}\right) \\
& =\lim _{\lambda \rightarrow \infty}\left[\frac{\lambda p_{\lambda}^{a}(a)}{w^{a}(a)}\left(L_{a \boldsymbol{a}}+\left(\lambda w_{\lambda}^{a}, h^{a}\right)\right)\right] .
\end{aligned}
$$

(48) then follows from (47).

In essence we have obtained by largely analytic methods quantitative expressions for the probabilistic influence of the $Q$-matrix on the process, in particular at $\zeta_{A}^{a}$. To get a better idea of the content of the Corollaries, the reader should apply them to the Feller-McKean $Q$-matrix described above and to the case when all states are stable, but not necessarily conservative $\left(\sum_{i \neq a} q_{a} \leqq q_{a}<\infty\right)$.

\section{The Construction Problem}

The problem of constructing a $P(t)$ on $E=I \cup A$ from a given $F(t)$ on $I$ and a given initial derivative matrix $Q$ on $E \times E$ can be "solved" in the sense that the analytic procedure used in the decomposition can be reversed. The unsatisfactory aspect is that very strong conditions on the functions $\left\{h^{a}, a \in A\right\}$ must be made. It is not difficult to define the construction for a mixture of ordinary and fictitious states by utilizing the constraint of Proposition 1, but in the spirit of the preceding results, we will content ourselves with the case when $A$ is to be composed of ordinary states. Our purpose is not really to give yet another construction (see e.g. Neveu [8]), but rather (i) to verify that the decomposition can in fact be reversed and (ii) to investigate the case when the $Q$-matrix uniquely defines an associated $P(t)$. In this regard we should mention that the recent papers of Williams [12] and Reuter [13] were motivating factors for our efforts here.

Let us assume that we are given $F(t)$ on $I$ such that $f_{i j}^{\prime}(0)=q_{i j}$.

$$
h^{0}(i) \equiv \lim _{t \rightarrow \infty} \sum_{I} f_{i j}(t)
$$

is invariant to $F(t)$. We assume that we are given $\left\{h^{a}(i), a \in A\right\}$ such that

(i) $\sum_{a \in A} h^{a}(i)=1-h^{0}(i)$

(ii) $h^{a}(i)=\int_{0}^{\infty} h_{i}^{a}(s) d s$ with $h_{i}^{a}(s) \geqq 0, \lim _{s \rightarrow 0} h_{i}^{a}(s)=q_{i a}$, and $h^{a}(s)$ a continuous exit law relative to $F(t)$. 
The remaining assumptions tie the $h^{a}(\cdot)$ to $q_{a} . F_{\lambda}$ denotes the Laplace transform as before.

(iii) $\sum_{i} q_{a i} f_{\lambda}(i, I)<\infty$ for $\lambda>0$,

(iv) $\sum_{j \neq a}^{i} q_{a j}\left(1-h^{a}(j)\right)<\infty$

(we follow the conventions of $h^{a}(c)=\delta_{a c}$ and $0 \cdot \infty=0$ ).

(v) If $q_{a}-\sum_{1 \neq a} q_{a 1}>0$, then there exists a null entrance law $v^{a}(t)$ such that

$$
\lim _{t \rightarrow 0} \sum_{i} v_{i}^{a}(t)=q_{a}-\sum_{1 \neq a} q_{a 1} .
$$

Theorem 5. Subject to (i)-(v) there exists a semigroup $P(t)$ on $E$ with initial derivative matrix $Q$. The resolvent of $P(t)$ is of the form

$$
p_{\lambda}(i, j)=f_{\lambda}(i, j)+\sum_{a} \sum_{b \in A} h_{\lambda}^{a}(i) \frac{p_{\lambda}^{a}(b) w_{\lambda}^{b}(j)}{w^{b}(b)}
$$

where all components have the same representation as in Theorem 1.

Proof. The technique used here is almost identical with that of [2] and [9], and we shall omit many of the details.

Let $\left\{V_{\lambda}^{a}, a \in A\right\}$ be the Laplace transforms of null entrance laws, subject to the constraints

$$
\lim _{\lambda \rightarrow \infty}\left(\lambda V_{\lambda}^{a}, 1-h^{a}\right)<\infty,
$$

the normalization assumption of (v) above and finally $V_{\lambda}^{a} \equiv 0$ if $a$ is stable and conservative. Define

Let

$$
\bar{w}_{\lambda}^{a}(i)=\sum_{I} q_{a j} f_{\lambda}(j, i)+V_{\lambda}^{a}(i) .
$$

define

$$
A_{0}=\left\{a: \sum_{b \neq a} q_{a b}+\lim _{\lambda \rightarrow \infty}\left(\lambda \bar{w}_{\lambda}^{a}, 1-h^{a}\right)=0\right\},
$$

and let

$$
w^{a}(a)^{-1}= \begin{cases}\sum_{\widetilde{E}} q_{a j}\left(1-h^{a}(j)\right)+\lim _{\lambda \rightarrow \infty}\left(\lambda V_{\lambda}^{a}, 1-h^{a}\right) & a \notin A_{0} \\ \left(\bar{w}_{1}^{a}, h^{a}\right) & a \in A_{0}\end{cases}
$$

As before

$$
w_{\lambda}^{a}(i)= \begin{cases}w^{a}(a) \bar{w}_{\lambda}^{a}(i) & i \in I \\ w^{a}(a) \delta_{a i} & i \in A .\end{cases}
$$

$$
L_{a b}= \begin{cases}\delta_{a b} & a \notin A_{0} \\ 0 & a \in A_{0},\end{cases}
$$

$\Omega(a, b)=0$ for $a \in A_{0}$ and otherwise

where

$$
\Omega(a, b)=\left(1-\delta_{a b}\right)\left(w^{a}(a) q_{a b}+U_{\infty}(a, b)\right)
$$

$$
U_{\lambda}(a, b)=\lambda\left(w_{\lambda}^{a}, h^{b}\right) .
$$

The proofs that $L-\Omega+U_{\lambda}$ is invertible and that

$$
P_{\lambda}=F_{\lambda}+\sum_{a} \sum_{b} h_{\lambda}^{a}\left(L-\Omega+U_{\lambda}\right)_{a b}^{-1} w_{\lambda}^{b}
$$


satisfies the resolvent equation and is stochastic follows as in [9]. The use of $w^{a}(a)>0$ is the only variation on the theme.

To verify that $\lambda P_{\lambda}(i, j) \rightarrow \delta_{i j}$ and that $Q$ is the initial derivative matrix we note that

$$
\begin{array}{ll}
\lambda h_{\lambda}^{a}(i) \rightarrow q_{i a} & i \in I, \\
\lambda w_{\lambda}^{a}(j) \rightarrow w^{a}(a) q_{a j} & j \in I, \\
\lambda^{2} p_{\lambda}^{a}(b) \rightarrow q_{a b} & a \neq b \in I, \\
\lambda\left(1-\lambda p_{\lambda}(a, b)\right) \rightarrow q_{a}, &
\end{array}
$$

and proceed by cases. The calculations are easy and involve at worst the use of Lemma 2.

Finally the existence of an associated $P(t)$ is guaranteed by Reuter [11: Section 1].

9.

We now have the machinery for both the decomposition and construction of $P(t)$ relative to $(I, A, Q, F(t))$ and are in a position to discuss uniqueness: when does a given $Q$-matrix have one and only one stochastic $P(t)$ associated with it? In general this will be the exception rather than the rule; however, by limiting the degree of freedom found in the construction we can obtain sufficient conditions:

Theorem 6. Suppose

(a) $q_{i}<\infty$ for $i \in I$.

(b) $\hat{F}(t)$ is stochastic where $\hat{F}(t)$ is the minimal process on $E \times E$ constructed from $\widehat{Q}$ :

$$
\hat{q}_{i j}= \begin{cases}q_{i j} & i \in I \\ 0 & i \in A .\end{cases}
$$

c) There exists no null entrance law $V(t)$ relative to $\hat{F}(t)$ on I with the property

for some $a \in A$.

$$
\lim _{t \rightarrow 0}\left(v(t),\left(1-\hat{f}_{\bullet_{a}}(\infty)\right)\right)<\infty
$$

Then if there exists a stochastic $P(t)$ with initial derivative matrix $Q$, it is unique.

Remark. The conditions for existence can be checked by using $h^{a}(i)=\hat{f}_{i a}(\infty)$. Note that assumption (c) forces $\sum_{j \neq i} q_{i j}=q_{i}$ for all $i \in A$, while the stochasticity of $\hat{F}(t)$ forces the same equality for $i \in I$.

Proof. Let $P(t)$ be decomposed as in Theorem 1. By definition $F(t) \geqq \widehat{F}(t)$ on $I \times I$. By examining the meaning of $h^{a}(i)$ and $\hat{f}_{i a}(\infty)$ and using the stochasticity of $\hat{F}(t)$, we have

By assumption (c),

$$
\begin{array}{cc}
f_{i j}(t)=\hat{f}_{i j}(t) & i, j \in I \\
h^{a}(i)=\hat{f}_{i a}(\infty) & i \in I .
\end{array}
$$

$$
w_{i}^{a}(t)=\left(\sum q_{a j} f_{j i}(t)\right) w^{a}(a),
$$

and the normalization of $w^{a}(a)$ (see e.g. the definitions in Theorem 5) show all components of the decomposition of $P(t)$ are expressible in terms of $Q$. $\quad \square$ 
In [12] Reuter treated the case when $Q$ is diagonal on $I \times I$ and $A=\{a\}$, where $a$ is instantaneous with $\sum_{j \neq a} q_{a j}=\infty$. As an application of Theorems 5 and 6 , we have the generalization: $j \neq a$

Corollary 1. Suppose $A$ is finite, $Q$ is diagonal on $I \times I$ and

(a) $0 \leqq \sum_{j} q_{i a_{j}}=q_{i}<\infty$ for $i \in I$

$$
0 \leqq \sum_{j} q_{a j}=q_{a} \leqq \infty \text { for } a \in A
$$

(b) Using the convention that $q_{j a} / q_{j}=0$, if $q_{j}=0$

$$
\sum_{E-a} q_{a j}\left(1-q_{j} / q_{j}\right)<\infty .
$$

(c) For $\lambda>0$

$$
\sum \frac{q_{a j}}{\lambda+q_{j}}<\infty
$$

Then there exists one and only one stochastic $P(t)$ with initial derivative matrix $Q$.

Proof. Existence follows from Theorem 5 with $f_{i j}(t)=\delta_{i j} e^{-q_{i} t}$ and $h^{a}(i)=q_{i a} / q_{i}$. Uniqueness follows from Theorem 6.

Finally we show that the analysis of this paper may be applied to a generalization of the case discussed by Williams [13].

Proposition 9. Suppose $A=\left\{a_{1}, a_{2}, \ldots, a_{n}\right\}$ is finite and

$$
\sum_{i=1}^{n} q_{a_{i} j} \geqq \delta>0
$$

for all $j \in I=E-A$. If there is a $P(t)$ with $Q$ as an initial derivative matrix then while

$$
\text { (c) } \sum_{j \neq i} q_{i j}=q_{i}<\infty \quad \text { for } i \notin A
$$

$$
\sum_{j \neq a} q_{a j}=q_{a}<\infty \quad \text { for } a \in A
$$

if $\left\{j: q_{a j} \geqq \delta / n\right\}$ is finite.

$$
\left(\mathrm{c}_{2}\right) \sum_{j} q_{a j} f_{\lambda}(j, I)<\infty,
$$

where $F_{\lambda}$ is the resolvent associated with the minimal process on $I \times I$.

If $A=\{a\}$, the converse assertion is true as well.

Proof. If $P(t)$ exists, $\left(\mathrm{c}_{2}\right)$ follows at once from the last exit decomposition relative to $A$ and the minimality of $F_{\lambda}$.

To prove $\left(\mathrm{c}_{1}\right)$ first decompose relative to $A$ and define $I_{i} \equiv\left\{j: q_{a_{i}} \geqq \delta / n\right\}$. Since for all $a_{i} \in A$

$$
\sum q_{a_{i j}}\left(1-h^{a_{i}}(j)\right)<\infty,
$$

$h^{a_{i}}(j)>\frac{1}{2}$ for all but a finite number of $j \in I_{a_{i}}$. Hence $I_{i} \cap I_{j}$ is finite for all $a_{i} \neq a_{j}$, and we may redefine them so that there is a null intersection.

From

$$
\infty>\sum_{j} \sum_{i=1}^{n} q_{a_{i} j} f_{\lambda}(j, I) \geqq \delta \sum_{j} f_{\lambda}(j, I),
$$


it follows that $f_{\lambda}(\cdot, I)$ is a finite measure. Hence the only null entrance law $v(t)$ with $v_{j}(t) \leqq M<\infty$ is $v(t) \equiv 0$. Suppose now that $I_{i}$ is finite. Then using the usual notation

$$
\begin{aligned}
w^{a_{i}}\left(a_{i}\right)^{-1} & \geqq \sum_{j} v_{j}^{a_{i}}(t)\left(1-h^{a_{i}}(j)\right) \\
& \geqq \sum_{j} v_{j}^{a_{i}}(t) \sum_{k \neq i} h^{a_{k}}(j) .
\end{aligned}
$$

But the last term on the right is $>\frac{1}{2}$ on all but a finite number of $j$. Hence $v_{j}^{a_{i}}(t) \equiv 0$ and from (43)

We also have

$$
\sum_{j \neq a_{i}} q_{a_{i j}}=q_{a_{i}} .
$$

and hence $q_{a_{i}}<\infty$.

$$
\sum q_{a_{i} j} \sum_{1 \neq i} h^{a_{1}}(j) \leqq w^{a_{i}}\left(a_{i}\right)^{-1},
$$

To prove the remainder of $\left(c_{1}\right)$ decompose relative to $A \cup \alpha$, where $\alpha$ is any state in $I$, and use similar reasoning to find $\sum_{j \neq \alpha} q_{\alpha j}<\infty$ and $v^{\alpha}(t) \equiv 0$.

The converse follows at once by defining $h^{a}=1-h^{0}$ and verifying the conditions preceding Theorem 5 .

\section{References}

1. Chung, K.L.: Markov chains with stationary transition probabilities, 2nd ed. Berlin-HeidelbergNew York: Springer 1967.

2. - On the boundary theory for Markov chains II. Acta math. 115, 111-163 (1966).

3. - Lecture notes on boundary theory for Markov chains. Princeton: Univ. Press 1970.

4. Doob, J.L.: Compactification of the discrete state space of a Markov process. Z. Wahrscheinlichkeitstheorie verw. Geb. 10, 236-251 (1968).

5. Feller, W., McKean, H.P., Jr.: A diffusion equivalent to a countable Markov chain. Proc. nat. Acad. Sci. USA 42, 351-354 (1967).

6. Kingman, J.F.C.: Linked systems of regenerative events. Proc. London math. Soc., III. Ser. 15, $125-150$ (1965).

7. Lamb, C.: Boundary theory for Markov chains. Thesis, University of Ill. (Urbana) (1968).

8. Neveu, J.: Lattice methods and submarkovian processes. Proc. Fourth Berkeley Sympos. math. Statist. Probab. 2, 347-391 (1960).

9. Pittenger, A.O.: Boundary decomposition of locally-Hunt processes. Advances in probability II. New York: Marcel Dekker (to appear).

10. Ray, D.: Resolvents, transition functions and strongly Markovian processes. Ann. of Math., II. Ser. 70, 43-72 (1959).

11. Reuter, G.E. H.: Denumerable Markov processes (II). J. London math. Soc. 37, 63-73 (1962).

12. - Remarks on a Markov chain example of Kolmogorov. Z. Wahrscheinlichkeitstheorie verw. Geb. 13, 315-320 (1969).

13. William, D.: A note on the $Q$-matrices of Markov chains. Z. Wahrscheinlichkeitstheorie verw. Geb. 7, 116-121 (1967).

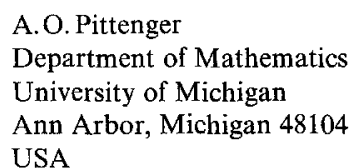

(Received July 1, 1970) 